\title{
Miranda
}

Revue pluridisciplinaire du monde anglophone /

Multidisciplinary peer-reviewed journal on the English-

speaking world

17 | 2018

Paysages et héritages de David Bowie

\section{Jean-Loup Bourget, Anne-Marie Paquet-Deyris, Françoise Zamour. Histoire, légende, imaginaire: nouvelles études sur le Western}

\section{Raphaëlle Costa de Beauregard}

\section{(2penEdition}

\section{Journals}

Édition électronique

URL : http://journals.openedition.org/miranda/14898

DOI : $10.4000 /$ miranda. 14898

ISSN : 2108-6559

\section{Éditeur}

Université Toulouse - Jean Jaurès

\section{Référence électronique}

Raphaëlle Costa de Beauregard, « Jean-Loup Bourget, Anne-Marie Paquet-Deyris, Françoise Zamour. Histoire, légende, imaginaire: nouvelles études sur le Western », Miranda [En ligne], 17 | 2018, mis en ligne le 02 octobre 2018, consulté le 16 février 2021. URL : http://journals.openedition.org/miranda/14898; DOI : https://doi.org/10.4000/miranda.14898

Ce document a été généré automatiquement le 16 février 2021.

\section{cc) (1)}

Miranda is licensed under a Creative Commons Attribution-NonCommercial-NoDerivatives 4.0

International License. 


\title{
Jean-Loup Bourget, Anne-Marie Paquet-Deyris, Françoise Zamour. Histoire, légende, imaginaire : nouvelles études sur le Western
}

\author{
Raphaëlle Costa de Beauregard
}

\section{RÉFÉRENCE}

Jean-Loup Bourget, Anne-Marie Paquet-Deyris, Françoise Zamour. Histoire, légende, imaginaire : nouvelles études sur le Western. Paris : Editions rue d'Ulm / Presses de l'Ecole normale supérieure, 2018, 146 p., ISBN : 978-2-7288-2781-7.

Dans son avant-propos (11-18) Jean-Loup Bourget rappelle que ce volume a pour thème le sujet de l'agrégation externe d'anglais 2018: «La construction de l'Ouest américain (1865-1895) dans le cinéma hollywoodien ». Le décalage entre le cinéma hollywoodien et la période de 1865 à 1895 est une difficulté qui incite la réflexion à se recentrer sur le concept de 'construction', et son caractère imaginaire, les films étant conditionnés par un corpus de légendes et/ou de récits historiques à propos de cette période pré-cinématographique. Les articles sont présentés comme le veut l'usage. Mais un détour par la table des matières fait apparaître que les articles sont d'abord méthodologiques, puis comparatifs, et enfin consacrés à une analyse fine d'un seul film. Le dernier article revient pour ainsi dire aux sources puisqu'il est consacré à la photographie, laquelle est antérieure à la période considérée.

Pour Christian Viviani, avec un article intitulé «Le western, mise en place d'un genre (1903-1960) : un héritage culturel » (19-28), il s'agit de préciser ce que l'on entend par 'western'. La critique de cinéma aurait glissé de l'adjectif dénotant des qualités du genre filmique telles que pittoresque, ou exotisme, au substantif qui désigne un ensemble de codes. On aurait par ailleurs une évolution de la pastorale à la veine 
réaliste, et, s'agissant de la figure de l'Indien, de l'Indien romantique à l'Indien meurtrier. Ce personnage apparait dès 1894 dans un très court métrage Edison, puis chez Pathé et Griffith. En outre, le déplacement de la production cinématographique du New Jersey à la côte californienne serait contemporain de cette évolution du personnage. Or les années 1950 voient renaître la vision positive de l'Indien avec, entre autres, les films d'Anthony Mann. Christian Viviani cite et parfois va jusqu'à présenter un très grand nombre de films peu connus dont la variété permet de préciser, tout en l'élargissant, ce que l'on entend aujourd'hui par 'western' comme figure de la construction de l'Ouest américain dans le cinéma hollywoodien.

Dans son article : "Transnationalisme, révisionnisme, historiographie : les nouveaux horizons du western » (29-40), Hervé Mayer s'intéresse aux différents courants de la critique de films que la très grand profusion et variété de films qualifiés de 'western' a suscité. Les auteurs étudiés vont des années 1950 jusqu’à nos jours. Hervé Mayer distingue dans ce vaste corpus trois lectures, nationalistes, évolutionnistes, mythiques, pour en exposer ensuite de manière détaillée les problématiques. Au sein de chacune de ces 'lectures', l'auteur discerne une articulation autour de polarités sémantiques; ainsi le nationalisme s'oppose au transnationalisme, l'évolutionnisme s'oppose au révisionnisme, tandis que la lecture mythique s'oppose à une approche historiographique du genre. Cet article très éclairant permet une synthèse des différentes 'constructions' par les critiques de cinéma de ce que serait l'Ouest américain comme objet des productions hollywoodiennes.

Avec l'article en anglais d' Anne-Marie Paquet-Deyris, «Framing Otherness in The Iron Horse and Little Big Man », (41-56), le lecteur de ce volume quitte les thèses générales pour suivre une méthode comparative. Il s'agit ici de comparer le personnage de l'étranger, Dave dans The Iron Horse et Jack dans Little Big Man, et des constructions culturelles telles que la race et le genre (huit illustrations). Le choix d'acteurs blancs pour interpréter des Indiens est déjà un aspect significatif de la problématique envisagée. John Ford donnerait une image pacifiste des Blancs et guerrière des Indiens. On peut ici regretter que le personnage de Deroux, le traître blanc qui se fait passer pour un Cheyenne dans le film de Ford, ne soit pas présenté par l'auteur de l'article tel qu'il est. En effet la lecture qui est proposée de cette supercherie est tout autre : il s'agit de voir en Deroux une caricature de l'Indien stéréotypé aux dépens de l'indianité, car cela permet de faire le lien avec Little Big Man. Dans ce second film, la problématique de l'Autre se double de celle, plus douloureuse, de la double descendance.

5 Pour Emmanuelle Delanoë-Brun, "Brides in red, white and blue: the politics of marriage in classical and revisionist westerns", (57-70) la comparaison inclut un plus grand nombre de films tels que The Iron Horse, Shane et Heaven's Gate, et The Ox-bow Incident. Les rôles féminins investissent les valeurs utopiques de la vie en communauté, de l'éducation des enfants tout en étant incarnés par des figures féminines modernes. L'exemple type serait Dallas dans Stagecoach, ou encore Ruby dans The Iron Horse, et Ella dans Heaven's Gate. A la fois éligibles pour le mariage et prostituées, ces femmes incarnent la femme émancipée moderne, et sont maîtresses des forces contraires de la société. L'alternative est la maîtresse d'école de My Darling Clementine ou de Cheyenne Autumn. L'épouse indienne est aussi un modèle de civilisation, dans Broken Arrow et Little Big Man, mais vouée à la mort. Il faut attendre 2010 Meek's Cutoff pour voir ces stéréotypes féminins perdre de leur importance symbolique. L'Ouest américain 
pourrait dès lors faire l'objet d'une 'construction' en tant qu'expérience de l'inconnu au quotidien.

Dans un article intitulé « Du rêve de conquête au territoire des conflits : esquisse d'un imaginaire géographique du western », (71-78) Françoise Zamour étudie l'utilisation du paysage pour imaginer un passé : il s'agirait de la création d'un 'paysage-image'. Cette construction repose sur une dimension documentaire : le lieu de la fiction est situé sur la carte, et porte un nom Nevada, Montana, Arizona. Or le paysage du western est une construction imaginaire qui possède ses propres caractéristiques. Ainsi The Iron Horse s'ouvre sur Lincoln imaginant un pays unifié d'Est en Ouest. La forme cinématographique privilégiée est le panoramique, associée à un montage où les lieux éloignés deviennent proches. Si ces deux écritures sont compréhensibles pour le spectateur, c'est que celui-ci y est habitué par le voyage en chemin de fer. Les personnages portent aussi la marque de l'imaginaire lorsqu'ils surgissent sans avertissement dans le paysage. Et enfin, l'alternance entre paysage sauvage et paysage civilisé est tout droit hérité de la Bible dont le texte s'appliquerait au paysage Américain tout autant que la 'destinée manifeste' du pionnier.

Puis vient un article intitulé « Ford en contexte : à partir de My Darling Clementine (La Poursuite infernale 1946) », (79-92, trois illustrations) par lequel Jean-Loup Bourget ouvre la voie à une série de chapitres consacrés à l'étude d'un western particulier. La genèse du film de John Ford est rappelée : deux films sur le sujet $(1931,1939)$ avaient déjà été réalisés à partir d'une même biographie, mais se présentant comme une fiction à partir de ce qui aurait été un roman. Jacques Tourneur a repris le sujet en 1952, suivi de trois autres films. Mais Ford se serait inspiré de The Virginian (1914) le roman et son adaptation à l'écran par Cecil B DeMille. Lyrisme et nostalgie se trouvent dans la chanson au titre éponyme, sans oublier les liens avec la peinture de Remington. D'autres échos tels que le paysage du western épique, Monument Valley, et la présence d'acteurs également consacrés par le genre, ont contribué au succès d'un 'western épique' qui faisait «émerger le 'féminin' de Wyatt Earp, associé à la politesse, au raffinement, à la culture » (91).

Gilles Menegaldo, quant à lui, contribue un article en anglais, « The Man Who Shot Liberty Valance : Convention, Subversion and Innovation ", (93-106). Il étudie un seul film très en détail, valorisant ainsi l'étude de cas pour ce qu'elle apporte à la compréhension de la problématique de la construction d'un motif culturel tel que l'Ouest américain. Le film est surtout connu pour la réécriture de la scène d'anthologie du genre, la confrontation finale entre le bon et le méchant suivie par la mort de ce dernier. Une deuxième version de la mort du méchant nous est donnée dans le film, où nous voyons qu'il est exécuté d'une balle dans le dos. Cette réécriture de la scène mythique s'inscrit dans un réseau complexe de jeux d'échos à des niveaux différents. L'addition d'une dimension politique au texte source se décline sous la forme de deux scènes d'élections. Les citations intertextuelles sont autant de clins d'œil à ce que fut le western, par exemple Stagecoach, sans oublier les acteurs dont John Wayne qui se plaint d'être privé de son aura. Même l'écriture du western classique, son décor de grands paysages, ou son montage alterné lors du 'shoot out', sont remis en cause par John Ford qui signe ici un film réflexif autant que révisionniste. Cette micro-analyse permet de comprendre le fonctionnement d'un genre tout d'abord comme construction puis comme réflexion sur cette construction. 
Céline Murillo, avec "Paysage de la révision dans Dead Man (Jim Jarmusch, 1995) ", (107-122, six illustrations), s'intéresse à un mode de construction de l'Ouest américain qui consiste à en déconstruire les codes. Au lieu des vastes étendues du paysage modèle, le film nous fait pénétrer dans une forêt, et l'utopie de la 'destinée manifeste' disparaît du même coup. La figure du trajet et du cavalier traversant des paysages au galop, qui a déjà disparu avec Little Big Man, manque dans Dead Man, ainsi que l'échelle de plans 'américains' traditionnels : les plans rapprochés en contre-plongée expriment la perte des repères des personnages. Les six illustrations qui accompagnent cet article soulignent l'écart entre les paysages des peintres et ceux du film de Jarmusch. Par exemple, le paysage du western est le théâtre d'embuscades et d'une utilisation tactique des lieux. Avec Dead Man cadrage et perspective sont sans lien avec l'action, sans compter le personnage plus mort que vif. En somme Dead Man construit un Ouest dans un paysage qui «se fait tactile pour l'œil et donne à ressentir la fragilité des corps » (120).

L'article de Didier Aubert, "L'authentique, l'intime et l'histoire: photographier les Indiens à l'âge du cinéma », (123-138, 5 illustrations) porte à la connaissance du lecteur un ensemble de réflexions sur un aspect de la construction de l'Ouest américain qui touche au sens même de construction. L'histoire de la photographie d'Amérindiens au long du $19^{\mathrm{e}}$ siècle débouche sur la naissance du cinéma alors même que s'opérait la disparition tragique de leur culture. Or ces photos, des portraits pour la plupart, montrent une construction culturelle par les photographes bien avant le cinéma. Dans ce modèle, se mêlent, à des degrés divers, le sentiment national, le commerce du divertissement et l'invention de modèles culturels. Des photographes tels que Gertrude Käsebier (1852-1934), Walter Mc Clintock (1870-1949) ou Joseph K. Dixon (1858-1929) ont joué un rôle tout aussi important qu'un Edward S. Curtis plus réputé. Didier Aubert ouvre le débat sur le lien avec le cinéma en conclusion: "contrairement à la photographie, où la présentation de soi reste presque toujours une négociation, le cinéma cantonne rapidement l'Indien, et pour longtemps, au rôle d'un homme qui chute de son cheval».

\section{INDEX}

Keywords : Native American, Hollywood cinema, classical, conventions, women, genre, gender, History, Indian, innovation, legend, marriage, manhood, myth, photography, revisionism, subversion, western

Mots-clés : amérindien, cinéma hollywoodien, classicisme, convention, femme, genre, histoire, Indien, innovation, légende, mariage, masculinité, mythe, photographie, révisionnisme, subversion, western 
AUTEURS

RAPHAËLLE COSTA DE BEAUREGARD

Professeur Emérite

Université de Toulouse 2-Jean Jaurès

r.costa-de-beauregard@orange.fr 